\title{
Efficient Interference Mitigation in mmWave Backhaul Network for High Data Rate 5G Wireless Communications
}

\author{
Jia Shi' ${ }^{1}$, Qiang Ni ${ }^{1}$, Claudio Paoloni ${ }^{1}$, Francois Magne $^{2}$ \\ ${ }^{1}$ Lancaster University, Lancaster, UK \\ ${ }^{2}$ When-AB S.A., Paris, France \\ Email: j.shi@lancaster.ac.uk, q.ni@lancaster.ac.uk, c.paoloni@lancaster.ac.uk,fmagne@when-ab.fr
}

How to cite this paper: Shi, J., Ni, Q., Paoloni, C. and Magne, F. (2017) Efficient Interference Mitigation in mmWave Backhaul Network for High Data Rate 5G Wireless Communications. Int. J. Communications, Network and System Sciences, 10, 170-180.

https://doi.org/10.4236/ijcns.2017.105B017

Received: April 8, 2017

Accepted: May 23, 2017

Published: May 26, 2017

\begin{abstract}
This paper investigates the performance of the $\mathrm{W}$ band millimeter wave (mmWave) backhaul network proposed by our EU TWEETHER project. We focus on the downlink transmission of the mmWave backhaul network, in which each of the hubs serves a cluster of base stations (BSs). In the considered backhaul network, available frequency resources are first allocated to the downlink links with the consideration of fairness issue. In order to mitigate interference in the mmWave backhaul network, each hub operates the proposed algorithm, namely cooperation and power adaptation (CPA). Our simulation results show that, the backhaul network with mmWave capabilities can achieve a significant better throughput performance than the sub- $6 \mathrm{GHz}$ ultra high frequency (UHF) backhaul network. Furthermore, our simulations also reveal that the proposed CPA algorithm can efficiently combat interference in the backhaul network.
\end{abstract}

\section{Keywords}

mmWave Communication, Backhauling, Interference Mitigation

\section{Introduction}

Traditional microwave based cellular networks are facing more and more challenges due to the exponential growth of data traffic. Shifting the communication spectrum from traditional microwave to millimeter wave (mmWave) is widely recognized as one of the best attractive solutions to future wireless communication networks demanding high-volume capacity. Moreover, mmWave small cell networks have been thought of as a promising approach to boost the coverage and rate of future cellular networks [1]. However, it is important and challeng- 
ing to design a backhaul network connecting to an explosive growing number of small BSs.

Wireless backhaul network with mmWave capabilities can be a promising candidate for future wireless communication systems, due to its advantages of high capacity, energy efficient, low transmission delay and low cost, etc. Most of the work about mmWave communication in the literature mainly focuses on channel modeling [2] [3], and its applicability for indoor/outdoor environment over traditional cellular networks in terms of the coverage and rate probabilities [4]. By contrast, limited studies, such as [5] [6] [7] [8], have investigated mmWave backhaul networks. The authors in [5] [6] have studied the resource allocation in heterogeneous backhaul network which consists of both wired fiber and mmWave wireless communications. In [6], the authors have investigated the heterogeneous backhaul network's performance in terms of packet delay, and have also shown the new backhaul technologies over the traditional wired only technologies. Recently, the authors in [5] have studied joint scheduling of radio access and mmWave and fiber backhaul in hybrid heterogeneous network with the capabilities of device-to-device transmissions. By contrast, the authors in [7] [8] have investigated mmWave only backhaul networks. In [7], they have only addressed energy consumption issue for a renewable energy powered mmWave backhaul network, and a Stackelberg game based pricing scheme is proposed to manage the traffic latency over the backhaul network. Massive MIMO aided mmWave backhaul network has been addressed in [8], where the hybrid preceding and combining scheme is proposed.

Against this background, in this paper, we investigate the performance of the W band mmWave backhaul network proposed by our EU TWEETHER project [9] [10]. We focus on the downlink transmission of the mmWave backhaul network, in which each of the hubs serves a cluster of base stations (BSs). In the considered backhaul network, the available frequency resources are allocated to the downlink links based on the greedy algorithm with the consideration of fairness issue. In order to mitigate interference in the mmWave backhaul network, each hub operates the proposed algorithm, namely cooperation and power adaptation (CPA). The proposed CPA algorithm can set up cooperative transmissions for the poor links; meanwhile, it also utilizes the strategy of power adaptation to reduce the strong interference generated by the links. A range of simulation results including the outage probability and sum rate are provided to evaluate the performance of the mmWave backhaul network. Our simulation results show that, our backhaul network with mmWave capabilities can significantly outperform the sub- $6 \mathrm{GHz}$ ultra high frequency (UHF) backhaul network in terms of the outage probability performance. Furthermore, our simulations also reveal that the proposed CPA algorithm can efficiently combat interference in the backhaul network.

The rest of the paper is organized as follows. Section 2 provides the system model and states the main assumptions. Section 3 describes the subband allocation method. Section 4 proposes the interference mitigation schemes. Section 5 
demonstrates the performance results. Finally, Section 6 summarizes the conclusions.

\section{System Models}

Our project, namely EU TWEETHER, proposes novel W band mmWave based heterogeneous wireless networks with high data rate distribution, spectrum- and energy-efficiency. The conceptual structure of the proposed network is shown in Figure 1. The network model proposed by EU TWEETHER consists of three tiers, which are infrastructure tier, backhaul tier and access tier. More specifically, in infrastructure tier, we can set up several terabits data links via fiber optics for the communications between the data centers and the point of presences (PoP) nodes over hundreds of kilometers. In backhaul tier, the $\mathrm{W}$ band $\mathrm{mm}$ Wave backhaul links are established for the communication between the PoP hub nodes and multiple BSs in access tier. Finally, access tier supports the communication from BSs to user terminals, where we apply sub-6GHz UHF communication links. In this paper, we will focus on investigating the achievable rate performance of the mmWave backhaul tier proposed by our EU TWEETHER project.

In this paper, we consider the downlink transmission of the mmWave backhaul network, which consists of $N_{h u b}$ hubs and $N_{b s}$ BSs. In order to capture the main characteristics of the network proposed by our EU TWEETHER project, we assume that, any two hubs are separated by a distance of $d_{0} \geq 3 \mathrm{~km}$, and the $N_{b s}$ BSs are uniformly distributed in the network. Furthermore, we also assume that the distance of a backhaul link is in the range of $1 \mathrm{~km} \leq d_{1} \leq 2 \mathrm{~km}$, and any two BSs are separated by a distance of $d_{2} \geq 0.6 \mathrm{~km}$. The mmWave backhaul transmissions in the network experience both the pathloss effects and the small-scale fading. To model pathloss effects including shadowing for a mmWave link, we employ the model of [11]

$$
L_{d B}(d)= \begin{cases}\rho+10 \alpha_{L} \log (d)+\chi_{L} & \text { if LoS } \\ \rho+10 \alpha_{N}+\chi_{N} & \text { if NLoS }\end{cases}
$$

where

$$
\rho=20 \log \left(\frac{4 \pi}{f_{c}}\right) .
$$

A mmWave communication link is assumed to be non-line-of-sight (NLoS) if the line segment joining the mmWave BS and the user is blocked by buildings.

Otherwise, the link is thought of as line-of-sight (LoS). In (2), $f_{c}$ is the car-

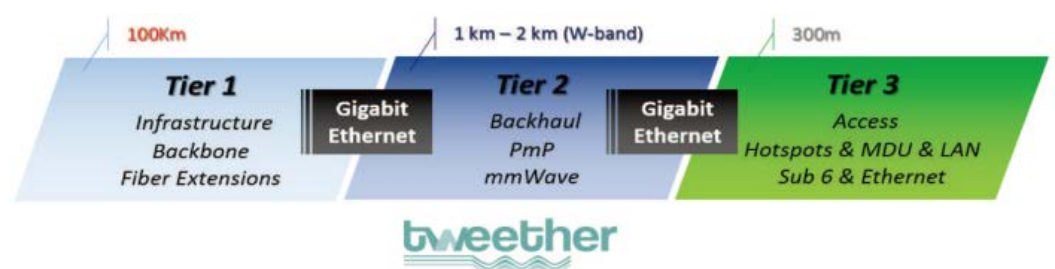

Figure 1. Conceptual network structure proposed by EU TWEETHER project. 
rier frequency at the mmWave frequency band, and $d$ represents the distance of a link. Furthermore, $\alpha_{L}$ and $\alpha_{N}$ are the pathloss exponents for LoS and NLoS cases respectively. $\chi_{L}$ and $\chi_{N}$ are shadowing effects, which are assumed to follow zero mean log normal distribution. We define the LoS probability of a mmWave link as $P_{L}(d)$ which is a decreasing function of the length of the communication link, and hence, the NLoS probability of a mmWave link is $P_{N}(d)=1-P_{L}(d)$. When a link becomes longer, it has a higher probability of being a NLoS link. Note that, the probability $P_{L}(d)$ can be characterized by blockage models according to various communication environments.

For the sake of theoretical study, we deploy the sectored antenna model to characterize the practical array patterns. Let us denote $g\left(M_{t}, m_{t}, \theta_{t}, \phi_{t}\right)$ and $g\left(M_{r}, m_{r}, \theta_{r}, \phi_{r}\right)$ as the sectored antenna patterns for a hub and a BS respectively. $M_{t}, M_{r}$ are the main lobe directivity gains, and $m_{t}, m_{r}$ are the back lobe directivity gains, while $\theta_{t}, \theta_{r}$ are the beamwidths of the antennas. Let the bore sight directions be $\phi_{t}$ and $\phi_{r}$, which are assumed to be 0 . For simplicity, we assume the antenna gain for a desired signal link is $G=M_{r} M_{t}$. However, each link may also have some interference links, and the antenna gain for an interference link is denoted as $\tilde{G}$, which can be given by

$$
\tilde{G}= \begin{cases}M_{r} M_{t}, & \text { with probability } p=\frac{\theta_{r} \theta_{t}}{4 \pi^{2}}, \\ m_{r} M_{t}, & \text { with probability } p=\frac{\left(1-\theta_{r}\right) \theta_{t}}{4 \pi^{2}}, \\ M_{r} m_{t}, & \text { with probability } p=\frac{\theta_{r}\left(1-\theta_{t}\right)}{4 \pi^{2}}, \\ m_{r} m_{t}, & \text { with probability } p=\frac{\left(1-\theta_{r}\right)\left(1-\theta_{t}\right)}{4 \pi^{2}} .\end{cases}
$$

In (3), we assume the beamwidth of an antenna, such as $\theta_{r}, \theta_{t}$, are independently and uniformly distributed in $(0,2 \pi]$.

In our mmWave backhaul network, each hub is able to transmit information to its serving BSs by using all spectrum of $B \mathrm{~Hz}$ available in $W$ band. Furthermore, we assume a hub communicates with its serving BSs based on orthogonal frequency division multiplexing (OFDM) employing $M$ subbands. Hence, in the downlink transmission of the backhaul network, the achievable rate of a BS served by its hub on a subband can be written as

$$
R_{n, m}^{(j)}=b_{m} \log _{2}\left(1+\gamma_{n, m}^{(j)}\right), j \in \mathcal{N}_{h u b}, n \in \mathcal{N}_{b s}^{(j)}, m \in \mathcal{M} .
$$

In (4), $b_{m}$ is the bandwidth of subband $m$. We define $\mathcal{N}_{\text {hub }}=\left\{1,2, \ldots, N_{\text {hub }}\right\}$ includes the indexes of hubs. $\mathcal{N}_{b s}^{(j)}$ contains the indexes of the BSs served by hub $j$, hence, we have $\sum_{j \in \mathcal{N}_{\text {hub }}}\left|\mathcal{N}_{b s}^{(j)}\right|=N_{b s}$. $\mathcal{M}$ includes the indexes of $M$ subbands available in the network. In (4), $\gamma_{n, m}^{(j)}$ is the signal-to-interferenceplus-noise rate (SINR) of BS $n$ served by hub $j$ on subband $m$, and it is defined by

$$
\gamma_{n, m}^{(j)}=\frac{P_{m}^{(j)} G_{n}^{(j)}\left(L_{n}^{(j)}\right)^{-1}\left|h_{n, m}^{(j)}\right|^{2}}{I_{n}+b_{m} \sigma^{2}} .
$$


In (5), $P_{m}^{(j)}$ is the transmission power of hub $j$ on subband $m$, and the transmission power for all subchannels are assumed to be the same. In addition to the large-scale fading effect, each communication link also experiences the small-scale fading, such as $h_{n, m}^{(j)}$, which is the independent Rayleigh fading channel between hub $j$ and BS $n$ on subband $m$. Furthermore, we assume that all the communication links have the same noise power, which is denoted by $\sigma^{2}$ in (5). Note that $I_{n}$ in (5) is the interference power that suffered by the transmission from hub $j$ to BS $n$.

In the considered mmWave backhaul network, each hub first needs to assign the available spectrum resource to the its serving BSs, in other word, each hub is required to allocate the $M$ number of subbands available to its BSs. However, the transmissions in the network will suffer from the interference generated by their co-subband links. Therefore, after subband allocation, we operate the proposed interference mitigation algorithm for the links suffering strong interference in order to improve the network's throughput performance. Let us now first discuss the subband allocation.

\section{Subband Allocation}

As mentioned in Section 2, a hub is assumed to employ all the available frequency bands to communicate with its serving BSs based on OFDM scheme. Therefore, hub $j$ needs to allocate $M$ subbands to its $\left|\mathcal{N}_{b s}^{(j)}\right|$ number of BSs, aiming to maximize the sum rate of the backhaul links.

In order to achieve the best trade-off between the performance and implementation complexity, each hub employs the greedy algorithm for subband allocation. In this paper, for simplicity and without loss of generality, the number of subbands available is larger than the number of BSs served by each hub, i.e. $M \geq\left|\mathcal{N}_{\text {hub }}^{(j)}\right|, \forall j \in \mathcal{N}_{\text {hub }}$. Based on the greedy algorithm, each hub allocates the $M$ subbands in $M$ iterations, in which during each a iteration the best available subband is assigned. Meanwhile, to achieve the best fairness of allocation, our allocation motivates to allocate each BS the same number of subbands. In this case, the subband allocation during a iteration can be described as

$$
\begin{gathered}
\mathcal{F}_{n^{*}}^{(j)} \leftarrow \mathcal{F}_{n^{*}}^{(j)} \cup m, \\
n^{*}=\max _{n \in \hat{\mathcal{N}}_{b s}^{(j)}}\left\{\frac{P_{m}^{(j)} G_{n}^{(j)}\left|h_{n, m}^{(j)}\right|^{2}}{L_{n}^{(j)} b_{m} \sigma^{2}}\right\}
\end{gathered}
$$

where $\mathcal{F}_{n}^{(j)}$ includes the indexes of the subbands assigned to BS $n$ served by hub $j . \quad \hat{\mathcal{N}}_{b s}^{(j)}$ contains the indexes of the BSs having the least number of subbands assigned. In this way, our subband allocation can ensure the best fairness in terms of allocation. After the subband allocation, the links using the same subband will cause interference to each other. Hence, the SINR of a communication link, such as the SINR in (5) for BS $n$ of hub $j$ on subband $m$, becomes

$$
\gamma_{n, m}^{(j)}=\frac{P_{m}^{(j)} G_{n}^{(j)}\left(L_{n}^{(j)}\right)^{-1}\left|h_{n, m}^{(j)}\right|^{2}}{\sum_{u \in \mathcal{N}_{h u b}, u \neq j} P_{m}^{(u)} \tilde{G}_{n}^{(u)}\left(L_{n}^{(u)}\right)^{-1}\left|h_{n, m}^{(u)}\right|^{2}+b_{m} \sigma^{2}}=\frac{P_{m}^{(j)} G_{n}^{(j)}\left(L_{n}^{(j)}\right)^{-1}\left|h_{n, m}^{(j)}\right|^{2}}{\sum_{u \in \mathcal{N}_{h u b}, u \neq j} I_{n, m}^{(u)}+b_{m} \sigma^{2}}
$$


where $\tilde{G}_{n}^{(u)}$ is given in (3). Known from (8), the through-put performance of the network may be significantly degraded by a communication link experiencing strong interference. To improve the network's performance, we need to operate interference mitigation to remove strong interference.

\section{Interference Mitigation}

For the sake of improving throughput performance of our mmWave backhaul network, in this section we propose a novel interference mitigation scheme, namely cooperation and power adaptation (CPA).

After the subband allocation discussed in Section 3, a desired communication link may suffer from strong interference imposed by other links using the same subband. Hence, the CPA algorithm is operated for the poor links, which either experience strong interference from their co-subband links, or generating strong interference to their co-subband links. Let us denote the set of the poor backhaul communication links on subband $m$ as $\Phi_{m}$, given by

$$
\begin{gathered}
\Phi_{m}=\hat{\Phi}_{m} \cup \check{\Phi}, \\
\hat{\Phi}_{m}=\left\{n \mid \eta_{n, m}^{(j)}<\eta_{t}, m \in \mathcal{F}_{n}^{(j)}, n \in \mathcal{N}_{b s}^{(j)}, \forall j \in \mathcal{N}_{h u b}\right\}, \\
\check{\Phi}_{m}=\left\{n \mid m \in \mathcal{F}_{n}^{(j)}, n \notin \hat{\Phi}_{m}, \forall j \in \mathcal{N}_{h u b}\right\}
\end{gathered}
$$

where $\eta_{t}$ is the signal-to-interference (SIR) threshold. In (9)-(11), $\eta_{n, m}^{(j)}$ is the SIR of the link, and it can be written as

$$
\eta_{n, m}^{(j)}=\frac{P_{m}^{(j)}\left(L_{n}^{(j)}\right)^{-1}\left|h_{n, m}^{(j)}\right|^{2}}{\sum_{u \in \mathcal{N}_{h u b}, u \neq j} I_{n, m}^{(u)}}
$$

where $I_{n, m}^{(u)}$ is given in (8).

The proposed CPA algorithm utilizes BS cooperation to mitigate interference in the backhaul network, in which the space time block coding (STBC) aided cooperative transmission can be established for the poor links. When the cooperation for the transmission to BS $n$ is set up by hub $j$ and hub $q$, the SINR in (8) for the link becomes

$$
\gamma_{n, m}^{(j)}=\frac{P_{m}^{(j)}\left(L_{n}^{(j)}\right)^{-1}\left|h_{n, m}^{(j)}\right|^{2}+I_{n, m}^{(q)}}{\sum_{q \in \mathcal{N}_{h u b}, u \neq j \neq q} I_{n, m}^{(u)}+b_{m} \sigma^{2}} .
$$

Known from (13), the achievable data rate of BS $n$ can be significantly improved when the interference $I_{n, m}^{(q)}$ imposed by hub $q$ is strong. In addition to cooperation, our CPA algorithm also employs the strategy of power adaptation. In that case, if a link suffers from strong interference, a hub may reduce the transmit power for the links, while guaranteeing the minimum service quality of the link over a range of at least $1 \mathrm{~km}$. By doing this, the links powered down will not cause strong interference to other links, and hence, the sum rate of the backhaul network will be improved. In this paper, our CPA algorithm aims to maximize the sum rate of the poor links, which can be expressed as 


$$
\mathcal{S}^{*}=\arg \max _{\mathcal{S}}\left\{\sum_{j \in \mathcal{N}_{h u b}} \sum_{n \in \Phi_{m}} R_{n, m}^{(j)}\right\}
$$

where $\mathcal{S}=\left\{s_{m}^{(j)}, m \in \mathcal{M}, j \in \mathcal{N}_{\text {hub }}\right\}$ contains the interference mitigation strategies. Specifically, $s_{m}^{(j)}=n$ means hub $j$ transmits information with the power $P_{m}^{(j)}$ to BS $n$ on subband $m$. By contrast, $s_{m}^{(j)}=0$ means that hub $j$ transmits information to $\mathrm{BS} n$ on subband $m$ with the reduced power $\beta P_{m}^{(j)}$, where $0 \leq \beta \leq 1$. Note that, the value of $\beta$ is related to the minimum data rate requirement and the minimum transmission range, which can be set up according to network requirements. Furthermore, in order to minimize the signaling burden, the hubs are only required to exchange binary interference information. For example, the binary interference information for $I_{n, m}^{(q)}$ in (13) can be defined by: $\hat{I}_{n, m}^{(q)}=1$ (strong interference) if $I_{n, m}^{(q)}>I_{t}$, otherwise $\hat{I}_{n, m}^{(q)}=0$ (small interference), where $I_{t}$ is the interference threshold.

Based on the optimization problem in (14), the principles of our CPA algorithm can be given as follows.

\section{Performance Results}

In this section, we provide a range of simulation results for demonstrating the achievable performance including the outage probability and sum rate of our backhaul network with the aid of $W$ band mmWave communication. Specifically, we also evaluate the performance of the proposed CPA algorithm in terms of mitigating interference in the network. For the sake of theoretical study, we assume that there are $N_{\text {hub }}=3$ hubs in the network, and for all simulations, the number of subbands is $M=N_{b s} / 3$. Furthermore, we assume that the noise power of each link is assumed to be the same, which is $\sigma^{2}$. The other simulation parameters are summarized in Table 1.

In Figure 2, we investigate the outage probability performance of the mmWave backhaul (BH) network, which is compared with the performance of the sub-6 GHz UHF only backhaul network. The outage probability is defined as the probability that the links in the network achieve the data rates below the minimum rate requirement $R_{\tau}$. For the UHF only backhaul network, we assume that the available bandwidth is $B=20 \mathrm{MHz}$ and the carrier frequency is $f_{c}=2.4 \mathrm{GHz}$. Without loss of generality, the other assumptions are the same as those for the mmWave backhaul network. Observed from Figure 2, the

Table 1. Simulation parameters for mmWave backhaul network.

\begin{tabular}{cccc}
\hline Parameter & Value & Parameter & Value \\
\hline$B$ & $1 \mathrm{GHz}$ & $\sigma^{2}$ & $-174 \mathrm{dBm} / \mathrm{Hz}$ \\
$f_{c}$ & $94 \mathrm{GHz}$ & $P_{m}^{(j)}$ & $46 \mathrm{dBm}$ \\
$M_{t}$ & $10 \mathrm{~dB}$ & $M_{r}$ & $10 \mathrm{~dB}$ \\
$m_{t}$ & $-10 \mathrm{~dB}$ & $m_{r}$ & $-10 \mathrm{~dB}$ \\
$\alpha_{L}$ & 2.09 & $\alpha_{N}$ & 3.34 \\
$\operatorname{Std}\left(\chi_{L}\right)$ & $5.0 \mathrm{~dB}$ & Std $\left(\chi_{N}\right)$ & $7.6 \mathrm{~dB}$ \\
\hline
\end{tabular}


mmWave backhaul network can significantly outperform the UHF network in all different minimum rate requirement scenarios. As shown, with the aid of the proposed CPA algorithm, the mmWave backhaul network can achieve a better outage probability performance, and a bigger performance gap can be observed in the range of $0.1 \mathrm{GHz} \leq R_{\tau} \leq 1 \mathrm{GHz}$. Furthermore, when the SIR threshold $\eta_{t}$ gets higher, the CPA algorithm can assist the network to achieve a higher performance. This is because the CPA algorithm operates interference mitigation for a larger number of communication links in the network.

In Figure 3, we show the outage probability of the mmWave backhaul network with and without employing the proposed CPA algorithm, when considering the different BS densities. First of all, we can see that, when the number of BSs gets bigger, the outage probability of the network gets higher. This observation implies that the backhaul communication links have higher probabilities of experiencing strong interference as the density of BSs served by the network increases. Second, our proposed CPA algorithm can significantly facilitate the network by efficiently mitigating interference. The performance gain of employing the CPA stays roughly the same for all different BS density scenarios. At last, once again, we observe that the outage probability becomes smaller as the SIR threshold gets bigger, in which more links are benefited from the CPA algorithm.

Finally, Figure 4 shows the effect of the thresholds $\eta_{t}$ and $I_{t}$ on the performance of the CPA algorithm. In the figure, we once more shows the significant performance improvement of the mmWave backhaul network by using the CPA to combat interference. As shown, the sum rate of the poor links increases as the SIR threshold $\eta_{t}$ or the interference threshold $I_{t}$ increases. This is because the CPA operates interference mitigation for more number of the poor links as the number of the poor links increases. However, in this case, it also

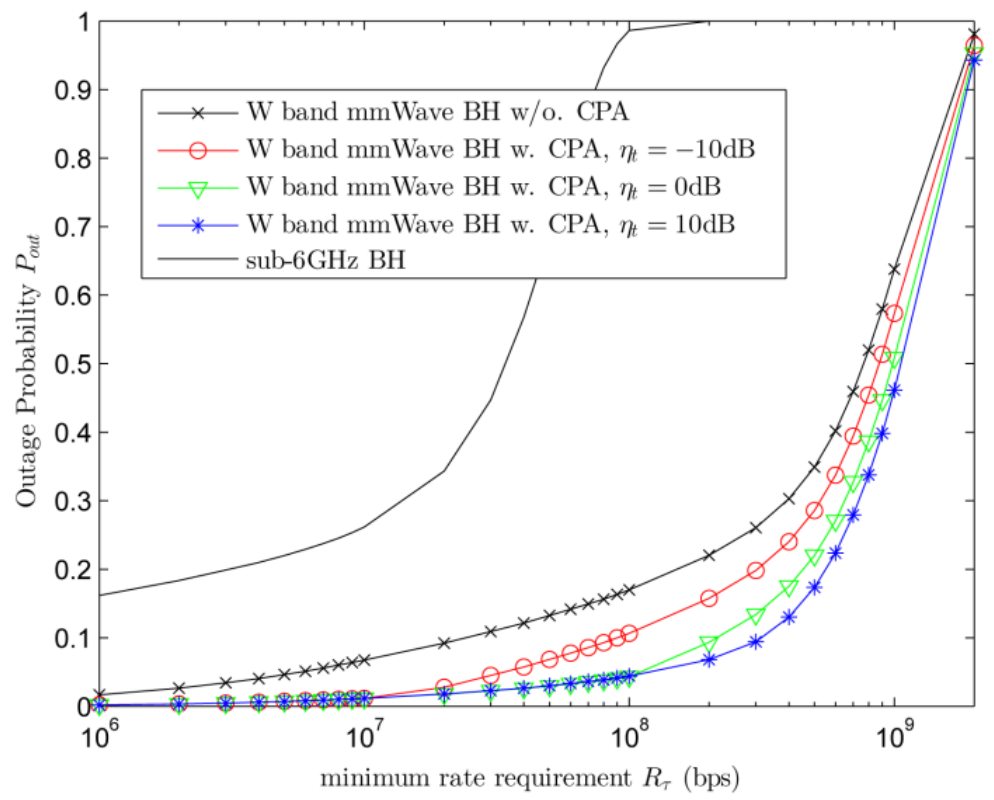

Figure 2. Outage probability of the backhaul networks, when employing various values of $R_{\tau}$ 
requires higher implementation complexity and cost. Therefore, it is important to choose proper SIR and ICI thresholds by jointly considering the system requirement, implementation complexity as well as desirable performance. From Figure 3 and Figure 4, we can conclude that, the proposed CPA algorithm can facilitate our mmWave back-haul network to achieve a better performance.

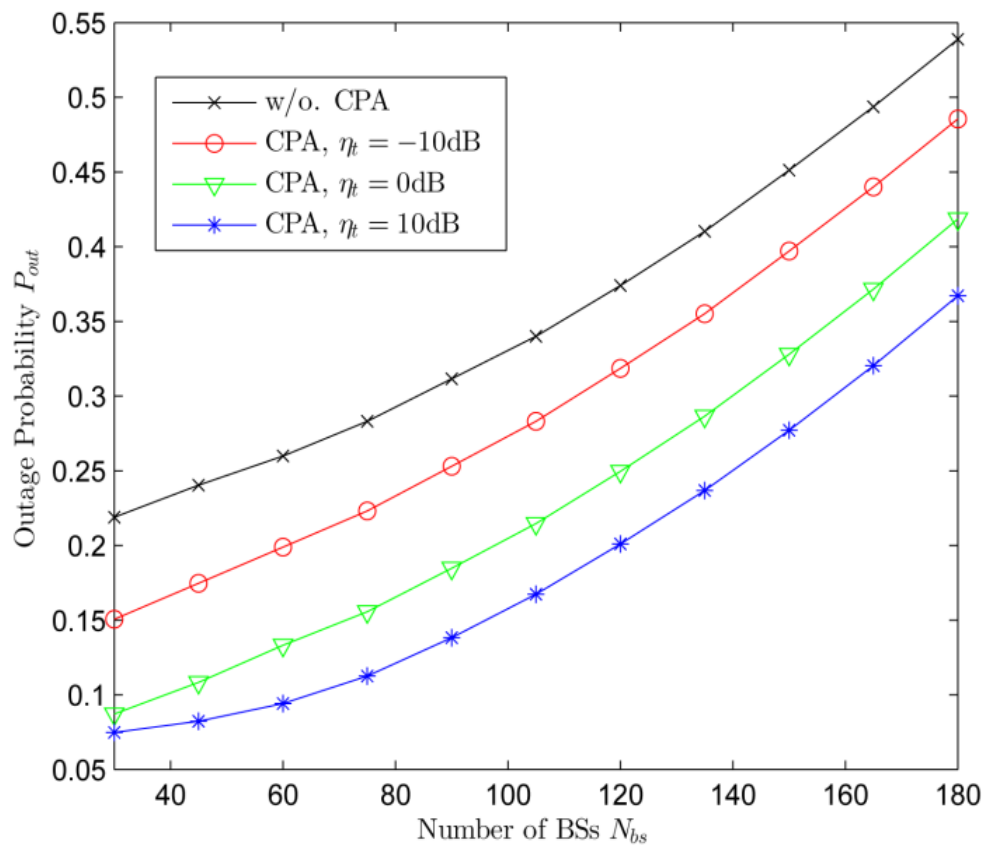

Figure 3. Outage probability of the $\mathrm{W}$ band mmWave backhaul networks having different densities of BSs, when assuming $R_{\tau}=0.3 \mathrm{Gbps}$.

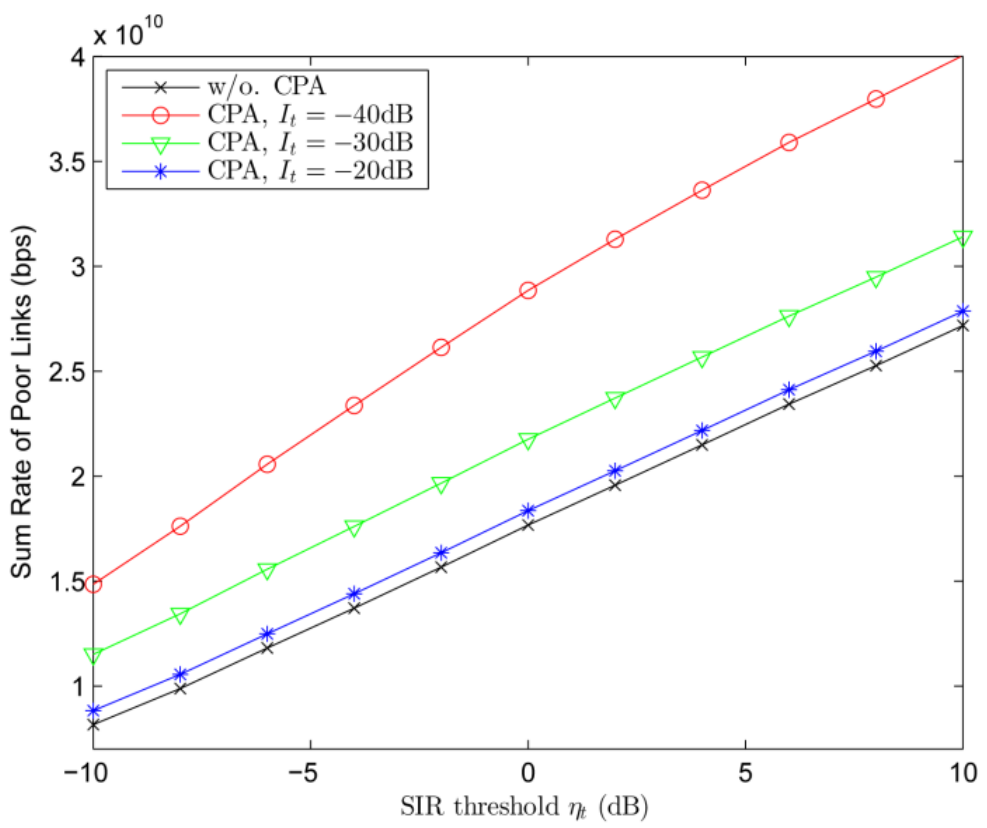

Figure 4. Sum rate of the poor links in the $\mathrm{W}$ band mmWave backhaul network employing the CPA algorithm under the various SIR threshold $\eta_{t}$ and ICI threshold $I_{t}$. 


\section{Conclusion}

In this paper, we have investigated the performance of the $\mathrm{W}$ band mmWave backhaul network proposed by our EU TWEETHER project. In order to improve the network's throughput performance, we have proposed the CPA algorithm to mitigate interference in the mmWave backhaul network. When the $\mathrm{CPA}$ algorithm is employed, the hubs are allowed to set up cooperative transmissions for the poor links, alternatively, the hubs are required to reduce the transmission power for some links that generate strong interference to other links. We have provided a range of simulation results including the outage probability and sum rate of the backhaul network. Our simulations have shown that, our mmWave backhaul network can significantly outperform the UHF backhaul network. Furthermore, our simulations have also implied that the proposed CPA algorithm can efficiently combat interference so that the backhaul network achieves a clear better performance. We conclude that the proposed CPA algorithm can be thought of as a promising solution for interference mitigation in our mmWave backhaul network.

\section{Acknowledgements}

This work is supported by EU H2020 TWEETHER project under grant agreement number 644678 .

\section{References}

[1] Grillo, G., Andrews, J., Buzzi, S., Choi, W., Hanly, S., Lozano, A., Soong, A. and Zhang, J. (2014) What Will 5G Be? IEEE J. Sel. Areas Commun., 32, 1065-1082. https://doi.org/10.1109/JSAC.2014.2328098

[2] Rappaport, T., et al. (2013) Millimeter Wave Mobile Communications for 5G Cellular: It Will Work! IEEE Access, 1, 335-349. https://doi.org/10.1109/ACCESS.2013.2260813

[3] Ragan, S., Rappaport, T.S. and Erkip, E. (2014) Milimeter-Wave Cellular Wireless Networks: Potentials and Challenges. Proceedings of the IEEE, 102, 366-385. https://doi.org/10.1109/JPROC.2014.2299397

[4] Bai, T. and Heath, R. (2015) Coverage and Rate Analysis for Millimeter-Wave Cellular Networks. IEEE Trans. Wireless Commun., 14, 1100-1114. https://doi.org/10.1109/TWC.2014.2364267

[5] Niu, Y., Gao, C., Li, Y., Su, L., Jin, D. and Vasilakos, A.V. (2015) Exploiting Device-to-Device Communications in Joint Scheduling of Access and Backhaul for mmWave Small Cells. IEEE J. Sel. Areas Commun., 33, 2052-2069. https://doi.org/10.1109/TWC.2014.2364267

[6] Zhang, G., Quek, T.Q.S., Kountouris, M., Huang, A. and Shan, H. (2016) Fundamentals of Heterogeneous Backhaul Design-Analysis and Optimization. IEEE Trans. Commun., 64, 876-889. https://doi.org/10.1109/TCOMM.2016.2515596

[7] Li, D., Saad, W. and Hong, C.S. (2016) Decentralized Renewable Energy Pricing and Allocation for Millimeter Wave Cellular Backhaul. IEEE J. Sel. Areas Commun., 34, 1140-1159. https://doi.org/10.1109/JSAC.2016.2520249

[8] Gao, Z., Dai, L., Mi, D., Wang, Z., Imran, M.A. and Shakir, M.Z. (2015) MmWave Massive-MIMO-Based Wireless Backhaul for the 5G Ultra-Dense Network. IEEE 
Wireless Communications, 22, 13-21. https://doi.org/10.1109/MWC.2015.7306533

[9] EU TWEETHER Project Website. https://tweether.eu/

[10] Paoloni, C., Letizia, R., Napoli, F., Ni, Q., Rennie, A., Zimmerman, R., Andre, F., Pham, K., Krozer, V., Magne, F., Burciu, I., Ramirez, A., Rocchi, M., Marilier, M. and Vilar, R. (2015) Horizon 2020 TWEETHER Project for W-Band High Data Rate Wireless Communications. IEEE 16th International Vacuum Electronics Conference (IVEC 2015), April 2015. https://doi.org/10.1109/ivec.2015.7223770

[11] Xu, X., Saad, W., Zhang, X., Xu, X. and Zhou, S. (2015) Joint Deployment of Small Cells and Wireless Backhaul Links in Next-Generation Networks. IEEE Communications Letters, 19, 2250-2253. https://doi.org/10.1109/LCOMM.2015.2494022

Submit or recommend next manuscript to SCIRP and we will provide best service for you:

Accepting pre-submission inquiries through Email, Facebook, LinkedIn, Twitter, etc. A wide selection of journals (inclusive of 9 subjects, more than 200 journals)

Providing 24-hour high-quality service

User-friendly online submission system

Fair and swift peer-review system

Efficient typesetting and proofreading procedure

Display of the result of downloads and visits, as well as the number of cited articles Maximum dissemination of your research work

Submit your manuscript at: http://papersubmission.scirp.org/

Or contactijcns@scirp.org 